\title{
Outpatient clinics for adults with congenital heart disease: increasing workload and evolving patterns of referral
}

\author{
M A Gatzoulis, S Hechter, S C Siu, G D Webb
}

\begin{abstract}
Objective-To examine the evolving role of specialised outpatient services for adult patients with congenital heart disease.

Design-A retrospective analysis of all patients attending the Toronto Congenital Cardiac Centre for Adults over three corresponding three month periods in 1987 , 1992, and 1997.

Setting-A tertiary referral centre.

Main outcome measures-Patient demographics, residence, medical and surgical history, type and source of referral, and investigations performed.

Results-In all, 570 patients were seen at the clinic during these three periods. There was a $44 \%$ and a $269 \%$ increase in workload between 1987 to 1992 and 1992 to 1997 , respectively. There was a steady fall in mean age of patients seen at the clinic with time $(38.5,33.6$, and 31.7 years in 1987, 1992, and 1997, respectively, p < 0.001). New referrals from community cardiologists and family physicians increased more in relative terms than did referrals from the Hospital for Sick Children, Toronto $(6.7 \%, 15 \%$, and $37.5 \%$, $\mathbf{p}=\mathbf{0 . 0 2}$ ). There was a steady increase in patients with previous reparative surgery $(48.9 \%, 59.2 \%$, and $69.2 \%, p<0.002)$. The proportion of patients with previous reoperations also increased $(2.3 \%, 10 \%$, and $9.2 \%, \mathrm{p}<0.01)$. Echocardiography remained the predominant method of diagnosis. The diagnostic mix did not change with time.
\end{abstract}

Conclusions-Over the past 10 years there has been a large increase in adults with congenital heart disease requiring and seeking specialised care in a tertiary health centre, with a concomitant evolution of referral patterns. These data may be helpful in planning of similar paediatric and adult cardiac services for this expanding population.

(Heart 1999;81:57-61)

Keywords: congenital heart disease; ambulatory clinics

Recent advances in surgical and medical management have dramatically altered the outlook for patients with congenital heart disease. ${ }^{1}$ As a result, ever increasing numbers of such patients survive to adulthood. Late complications (for example, exercise intolerance, arrhythmias, and sudden death) are not uncommon even among patients who previously underwent reparative surgery. Longitudinal follow up is thus recommended. ${ }^{23}$ Furthermore, there are patients with congenital heart disease who present beyond childhood, either because of poor access to specialised paediatric services or delayed diagnosis. It has been suggested that physicians assuming responsibility for adult patients with or without previous interventions for congenital heart disease require special expertise. ${ }^{4}$ Ready access to diagnostic, surgical, and other multidisciplinary facilities is also essential. ${ }^{6}$ The Toronto Congenital Cardiac Centre for Adults (TCCCA) was established in 1959 at the Toronto General Hospital (now The Toronto Hospital) to provide continuing care for adults with congenital heart disease originally cared for by the neighbouring Hospital for Sick Children, Toronto. The TCCCA has since evolved into one of the largest specialised units in the world for this expanding patient population. ${ }^{8}$ Its nucleus is the ambulatory clinic with direct access to on-site medical, surgical, and other multidisciplinary facilities. The purpose of our study was to examine the patterns of referral and the type and volume of workload of the TCCCA ambulatory clinic over the last decade, and define the evolving role of specialised outpatient services for adult patients with congenital heart disease.

\section{Methods}

The ambulatory TCCCA clinic at the Toronto Hospital is led by cardiologists (adult or paediatric) with expertise in the management of adult congenital heart disease. The clinics are now held twice weekly (once a week in 1987, with additional clinic days added in 1992 to accommodate increasing volumes of patients), and referrals are accepted from physicians primarily from the province of Ontario, but also from other regions of Canada and internationally. For the purpose of the study, referral sources were classified as: family physicians and community cardiologists, academic cardiologists (employed at a university hospital), and paediatric cardiologists. New referrals are booked into the next available clinic. Investigations for patients returning for routine follow up are booked at their preceding visit to the clinic according to a lesion targeted protocol. Additional investigations are ordered when clinically indicated. For new patients basic investigations are requested at their first visit. Since 1992, complex cases or cases presenting management dilemmas are discussed at the weekly conference. These conferences are routinely attended by cardiologists, cardiovascular 
Table 1 Clinic profile for the three three-month periods (March to May)

\begin{tabular}{lccrl}
\hline & 1987 & 1992 & 1997 & p Value \\
\hline Number of clinic days & 13 & 19 & 23 & \\
Number of patients & 90 & 130 & 350 & \\
New referrals & 16.7 & 15.2 & 13.9 & NS \\
$\quad$ Percentage of total number of patients & 6.7 & 15 & 37.5 & 0.02 \\
$\quad$ Community cardiologists/family physicians (\%) & 20 & 5 & 18.7 & NS \\
$\quad \begin{array}{l}\text { Academic cardiologists } \\
\quad \text { Paediatric cardiologists }\end{array}$ & 73.3 & 80 & 43.7 & 0.16 \\
$\begin{array}{l}\text { Conference } \\
\quad \text { Percentage of total number of patients }\end{array}$ & - & 6.9 & 5.4 & NS \\
Interventions & 16.7 & 14.6 & 12.6 & NS \\
$\quad$ Percentage of total number of patients & & &
\end{tabular}

Academic cardiologists, employed at a university hospital; Conference, percentage of cases referred for conference discussion from those seen at the clinic; Interventions, percentage of cases in which an interventional procedure followed the clinic visit.

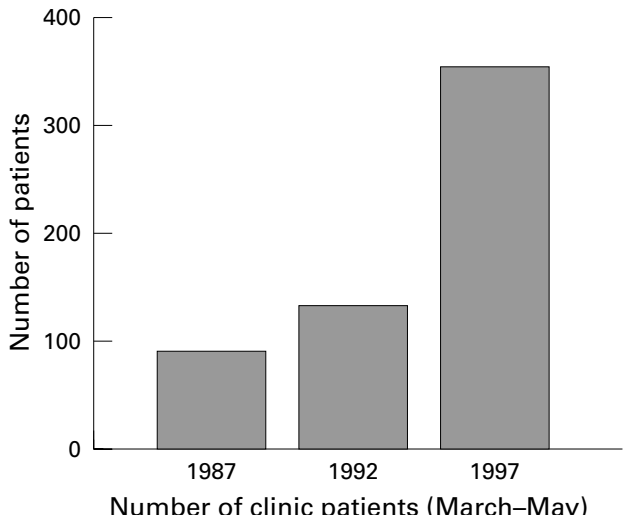

Figure 1 Patients seen at the TCCCA clinic during the same three month period in 1987, 1992, and 1997. Notice $44 \%$ and $269 \%$ increase between 1987 and 1992, and 1992 and 1997, respectively.

surgeons, respirologists, clinical psychologists, educational specialists, and postgraduate trainees. Cases discussed have either been seen at a recent TCCCA clinic or are referred from elsewhere for review. The meetings encourage

Table 2 Clinic patient characteristics for the three three-month periods (March to May)

\begin{tabular}{|c|c|c|c|c|}
\hline & 1987 & 1992 & 1997 & $p$ Value \\
\hline Mean (SD) age (years) & $38.5(8.9)$ & $33.6(13.5)$ & $31.7(11.7)$ & $<0.001$ \\
\hline Male $(\%)$ & 51.1 & 46.9 & 55.7 & NS \\
\hline Unoperated & 35.5 & 33.8 & 26.9 & 0.2 \\
\hline Palliation only & 16.7 & 19.3 & 18 & NS \\
\hline Previous repair & 48.9 & 59.2 & 69.2 & 0.002 \\
\hline Previous reoperation & 2.2 & 10 & 9.2 & 0.01 \\
\hline Pacemaker & 1.1 & 1.5 & 3.7 & NS \\
\hline \multicolumn{5}{|l|}{ Main diagnoses (\%) } \\
\hline Atrial/ventricular septal defect & 17.8 & 21.6 & 22 & NS \\
\hline Tetralogy of Fallot & 20 & 16.2 & 14.3 & \\
\hline LVOT obstructive lesions & 21.1 & 12.3 & 12 & \\
\hline Complete TGA & 7.8 & 7.7 & 10 & \\
\hline RVOT obstructive lesions & 9 & 6.2 & 7.7 & \\
\hline Coarctation of the aorta & 5.6 & 6.2 & 6.9 & \\
\hline Marfan syndrome & 2.2 & 6.2 & 5.4 & \\
\hline Congenitally corrected TGA & 2.2 & 3.1 & 4.3 & \\
\hline Atrioventricular septal defect & 1.1 & 1.6 & 3.4 & \\
\hline Eisenmenger syndrome & 1.1 & 1.6 & 3.1 & \\
\hline Ebstein anomaly of TV & 2.2 & 3.1 & 2.3 & \\
\hline Complex/other lesions & 8.9 & 19.2 & 12.9 & \\
\hline \multicolumn{5}{|l|}{ Investigations (\%) } \\
\hline Echocardiography & 44.4 & 39.2 & 55.7 & NS \\
\hline Holter monitoring & 7.8 & 7.7 & 6.9 & NS \\
\hline Exercise RNA & 6.7 & 3.1 & 2.9 & 0.06 \\
\hline Cardiac catheter (diagnostic) & 2.2 & 0.8 & 0.3 & NS \\
\hline Other & 1.1 & 2.3 & 4.6 & NS \\
\hline
\end{tabular}

Palliation only, for patients with one or more previous palliative, not "corrective," procedures; Previous repair, for patients who had undergone interventions, providing haemodynamic and or anatomical repair; Previous reoperation, for patients who had required further intervention, following previous repair. Previous repair and reoperation are not mutually exclusive - that is, the total is $>100 \%$.

L(R)VOT, left (right) ventricular tract obstruction; RNA, radionuclide angiography TGA, transposition of the great arteries; TV, tricuspid valve. consensus management decisions within the group, and at the same time, form part of the continuing education of the participants.

To profile the last 10 years, all patients who attended the TCCCA clinic during a three month period (March to May) in 1987, 1992, and 1997 were identified from the unit's computerised database and included in our study. We chose this period (March to May) to include the latest clinic cohort from 1997 in relation to the timing of the study. We also reviewed cases discussed at the unit's conference rounds during the same three month periods in 1992 and 1997. Demographics, residence, medical and surgical history, type and source of referral, investigations, referral for discussion at the conference rounds, and ensuing interventions were obtained from the hospital case notes and recorded for all patients. When multiple cardiac defects were present, diagnostic categorisation was made according to the predominant lesion. We elected to list only the commonly used diagnostic tests during the reported 10 year period. Routinely performed ECGs and chest radiographs were excluded from the analysis. Tests such as magnetic resonance imaging and cardiopulmonary exercise testing were included under a separate category of "others," because of the small number performed in the early part of the study. Operative history was classified as: unoperated, for patients with no previous interventions; palliative, for patients with one or more previous palliative, but no reparative, procedures; repair, for patients who had undergone interventions (surgical in the vast majority), providing haemodynamic and/or anatomical "correction" (that is, including the Mustard and Fontan procedures); and reoperation, for patients who had required further intervention, following previous repair. Results are presented as absolute numbers, percentages or mean (SD).

\section{STATISTICAL ANALYSIS}

We used the $\chi^{2}$ test to compare parametric variables between the three groups. For continuous variables a non-parametric test (Kruskal-Wallis test) or one way analysis of variance with Bonferroni correction for multiple comparisons was used (SPSS statistical package).

\section{Results}

\section{CLINICS}

The total number of patients seen at the clinic during these three three-month periods was 570 (table 1). There was a $44 \%$ and a $269 \%$ increase in workload between 1987 and 1992, and 1992 and 1997, respectively (fig 1). There was also an increase in the number of patients seen per clinic in 1997 (average 15.2 patients/ clinic in $1997 v 6.9$ in 1987 and 6.8 in 1992). The 350 study patients from March to May 1997 account for $22.8 \%$ of the total number of patients seen during the 12 month period between July 1996 and June 1997 at the TCCCA (1532 patients), a percentage which was not significantly different from the three month periods in 1987 and 1992 (551 patients 


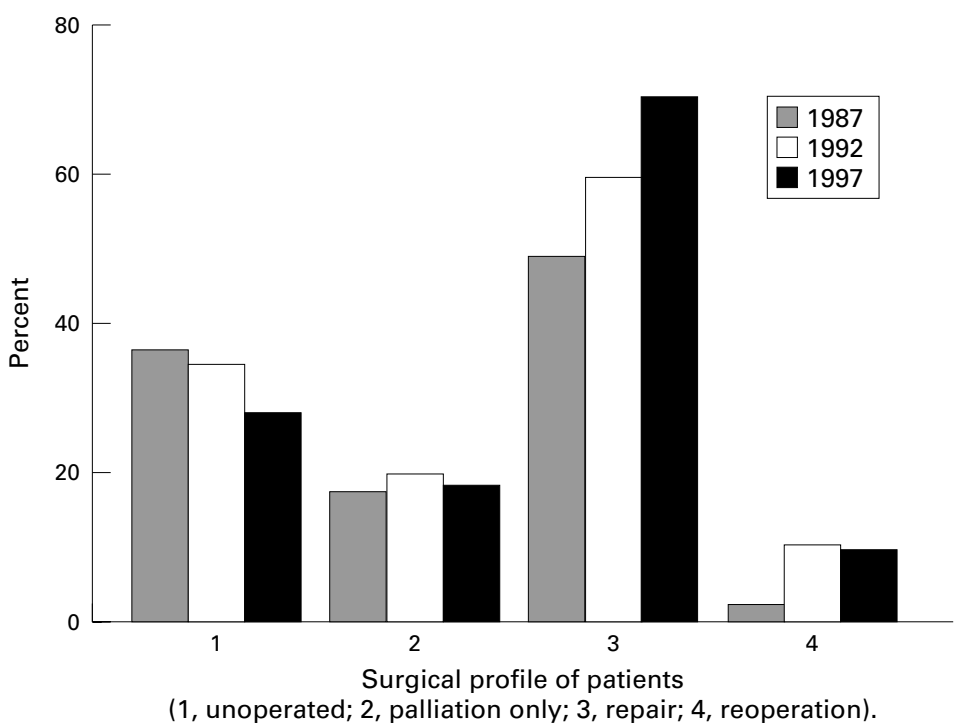

Figure 2 Surgical profile of patients at the time of their clinic visit. Notice significant increase between 1987 and 1997 in the percentage of patients with previous reparative surgery and in those who required reoperations after initial repair.

Table 3 Conference profile for two three-month periods (March to May)

\begin{tabular}{|c|c|c|c|}
\hline & 1992 & 1997 & $p$ Value \\
\hline Number of conferences & 11 & 9 & \\
\hline Number of cases discussed & 17 & 30 & \\
\hline Mean (SD) age of patients (years) & $31.6(14.2)$ & $38.8(14.7)$ & NS \\
\hline Male (\%) & 41.2 & 56.7 & NS \\
\hline \multicolumn{4}{|l|}{ Residence (\%) } \\
\hline Metro Toronto & 11.8 & 16.7 & NS \\
\hline Other Ontario & 82.4 & 56.7 & NS \\
\hline Outside Ontario & 5.9 & 26.7 & 0.08 \\
\hline \multicolumn{4}{|l|}{ Previous operations (\%) } \\
\hline Unoperated & 35.3 & 43.3 & NS \\
\hline Palliation & 23.5 & 10 & NS \\
\hline Repair & 41.2 & 53.3 & NS \\
\hline Reoperation & 11.8 & 3.3 & NS \\
\hline \multicolumn{4}{|l|}{ External referrals (\%) } \\
\hline Percentage of total number of cases & 52.9 & 46.7 & NS \\
\hline Community cardiologists & 44.4 & 50 & NS \\
\hline Academic cardiologists $\star$ & 33.3 & 42.9 & NS \\
\hline Paediatric cardiologists ${ }^{\star}$ & 22.2 & 7.1 & NS \\
\hline \multicolumn{4}{|l|}{ Outcome (\%) } \\
\hline No intervention & 11.8 & 23.3 & NS \\
\hline Additional data requested & 52.9 & 33.3 & NS \\
\hline Admission for intervention & 35.3 & 43.4 & NS \\
\hline
\end{tabular}

Additional data requested, additional information required before firm management recommendations could be made; Admission for intervention, case scheduled for elective intervention either at the tertiary or referring centre; External referral, new cases either seen at the clinic for the first time or referred directly for conference review; the remainder are follow up cases previously seen at the TCCCA; No intervention, no need for intervention at present.

${ }^{\star}$ Percentage in relation to external referrals. Repair and reoperation not mutually exclusive- that is, total is $>100 \%$.

$(17.6 \%)$ and 661 patients $(19.6 \%)$, respectively), suggesting that these samples are representative of the annual clinic workload.

There was a change in the age distribution of patients seen in the three different periods (table 2). There was a steady fall in mean age of patients seen at the clinic with time. The majority of patients were from the province of Ontario, with no significant differences seen in this pattern over the 10 year period, indicating that the overall referral population has not changed during the study period. The percentage of new referrals to the clinic compared with patients who were followed up remained stable. However, their absolute numbers were noticeably increased. The referring sources are shown in table 1 . There was a relative increase in new referrals from community cardiologists and family physicians, compared with new referrals from paediatric and academic cardiologists.

There were no significant differences in overall diagnoses between the three study periods (table 2). However, there was a large increase in patients with Marfan syndrome between 1987 and 1992, maintained in 1997. This is because of the TCCCA decision to include heritable cardiovascular disorders in its mandate. There were significant differences between the three periods in the number of patients with previous repair and in patients who had reoperations (table 2). There was a steady increase in patients with previous reparative surgery, with a concomitant fall in mean age, most probably suggesting that increasing numbers of such patients who had undergone repair for congenital heart disease are now followed up in our facility (fig 2). Not surprisingly, the incidence of reoperations has significantly increased since 1987, in keeping with progressive residual haemodynamic problems requiring reintervention. Trends in diagnostic tests are shown in table 2 . As expected, echocardiography was and has remained the predominant diagnostic method.

\section{CONFERENCES}

More cases were discussed in conferences in 1997 (table 3), in keeping with the increased volume of clinic patients. The number of cases discussed at each conference was also increased (3.3 cases/conference in $1997 v$ $1.5 /$ conference in 1992). There were no significant differences in age, sex, diagnoses, and previous interventions between the two groups. There was a trend towards an increase in the number of cases referred from outside the province of Ontario. Finally, there were no differences in referral sources or outcome with time.

\section{Discussion}

This study shows a pronounced increase over the past decade in the number of patients seen at the ambulatory clinic of one of the oldest and largest specialised units for adult congenital heart disease. This increase reflects primarily the growing numbers of patients with congenital heart disease who, having survived early surgery, enter adulthood. ${ }^{9}$ Improved awareness of this expanding field within the profession has played an additional role. It has been suggested that these patients' special needs are best met by a multidisciplinary approach in centres with adequate volumes both for developing and maintaining the necessary expertise. ${ }^{4510}$ Not surprisingly, once such services are established and become known to potential users a workload increase follows. ${ }^{11}$ More community based physicians, for example, referred their patients to our clinic in 1997 than 10 years before. A direct line of communication between potential referral sources and the specialised centre, coordination with clear follow up plans, and unrestricted access to the specialised facilities are all essential for the success of such services. ${ }^{9}$ Patients on the other hand find it reassuring to be followed up periodically in centres with concentrated expertise, 
where not only their medical needs are met, but also advice on important issues such as contraception, ${ }^{12}$ pregnancy, ${ }^{13-15}$ employment, and insurability ${ }^{16}$ is available. The increasing number of patients seen at the TCCCA with a decreasing mean age over time must be a reflection of all these factors.

There have been significant changes in the surgical profile of patients seen at the clinic between 1987 and 1997. More patients (in relative terms) had undergone reparative surgery. This is in keeping with the current thinking of repairing early rather than palliating congenital heart disease, whenever possible. Furthermore, the incidence of reoperations following previous repair has increased since 1987. This is in agreement with the recent report by Dore et al from the Royal Brompton Hospital in London, UK, ${ }^{17}$ in which reoperations represented the largest burden on their cardiac surgical services between 1991 and 1994 for a similar population. These data clearly underscore the need for continuous follow up of adult patients with congenital heart disease, as residual haemodynamic problems often progress with time, and may necessitate repeat operations late after initial repair. Not all paediatric patients with congenital heart disease require specialised follow up in adulthood but most do. ${ }^{10}$ Our view, and the view of others, ${ }^{156910}$ is that with the exemption of "benign" lesions - like mild pulmonary valve stenosis and postoperative atrial and ventricular septal defects-most patients with congenital heart disease would benefit from specialised follow up throughout their lives. The level of such supervision of course varies and depends by and large on the type of lesion. ${ }^{10}$

There was an increase in patients with Marfan syndrome, a result of the unit's policy to include this group with special needs. With further advances in fields such as molecular biology, other groups of patients with heritable cardiovascular disease-such as patients with hypertrophic cardiomyopathies-may benefit from a similar approach.

Echocardiography, both transthoracic and transoesophageal, was the predominant diagnostic test for our patients. Other modes, such as magnetic resonance imaging, are now establishing their firm role in areas of congenital heart disease. However, the numbers from this study were too small for a meaningful analysis. Ongoing developments in various non-invasive diagnostic techniques have also resulted in a more selective use of diagnostic cardiac catheterisation.

The conference meetings have gained popularity over the last five years, as illustrated by the increased number of cases discussed. They represent an open forum where complex adult congenital heart disease cases are discussed in depth among relevant disciplines, allowing for consensus management decisions. At the same time, they have an educational role for professionals from the group itself and perhaps from other centres, when difficult cases are referred for review. Our data show an interest among colleagues caring for adult congenital heart disease from other centres in this service. The need for free flow of information between professionals in this relatively new subspecialty can not be overemphasised. Further advances in this field are expected with the establishment of national and international networks for adult congenital heart disease. ${ }^{10}$ In addition, current developments with the internet and electronic mail will inevitably enhance this communication worldwide, and facilitate further such exchange of information.

With continuing advances in surgical, transcatheter, and medical management in congenital heart disease, further growth in the specialty of adult congenital heart disease is expected. The establishment of new specialised services for adult congenital heart disease in tertiary referral centres will serve this clinical need, ${ }^{6-8}$ and at the same time enhance research opportunities, education, and the smooth transition of care from paediatric to adult medicine. ${ }^{9}$ Longitudinal follow up data from such centres will facilitate necessary modifications of early management of congenital heart disease, aiming for an improved and sustained long term outcome for these patients. Concentrating on this field in an organised manner will also promote training of a new category of subspecialists from cardiology, cardiovascular surgery, paediatric cardiology, and other health professions alike. ${ }^{4}$ Sadly, in many parts of the world patients with congenital heart disease who outgrow the paediatric cardiac clinics are either "lost to follow up" or managed exclusively by physicians who are not experienced in congenital heart disease. Structured transitional programmes for these patients are clearly required to maintain early benefits from intervention for congenital heart disease. Close collaboration between adult units and their paediatric cardiac counterparts is essential for maximising the potential gains from such endeavours. ${ }^{18}$ Successful examples could function as raw models for other forms of congenital or chronic childhood disease, whereas smooth transition and continuation of care from paediatric to adult medicine may also be required.

We conclude that over the last decade there has been a large increase in adult patients with congenital heart disease requiring and seeking specialised care, with a concomitant evolution of referral patterns to our tertiary referral centre. Further expansion of this field can be expected. Our data may be helpful in future planning of similar paediatric and adult cardiac services for these patients.

We acknowledge our colleagues from the Hospital for Sick Children, Toronto for their continuing support of this programme, as well as hospital and community physicians for sharing patient care, and the TCCCA medical, nursing, and administrative staff for their commitment to the field of adult congenital heart disease. MAG was supported in part by a 1997
University of Toronto, Department of Medicine Postgraduate University of Toro
Fellowship Award

1 Perloff JK. Pediatric congenital cardiac becomes a postoperative adult. The changing population of congenital heart disease. Circulation 1973;47:606-19.

2 Somerville J. Management of adults with congenital heart Somerville J. Management of adults with congenital heart
disease: an increasing problem. Annu Rev Med 1997;48: 283-93.

3 Perloff JK. Adults with surgically treated congenital heart disease. Sequelae and residua. $\mathcal{F} A M A$ 1983;250:2033-6. 
4 Skorton DJ, Cheitlin MD, Freed MD, et al. Task Force 9: Skorton DJ, Cheitlin MD, Freed $\mathrm{MD}$, et al. Task Force 9: training in the care of adult patients with
disease. 7 Am Coll Cardiol 1995;25:31-3.

5 Somerville J. The physician's responsibilities: residua and sequelae. F Am Coll Cardiol 1991;18:325-7.

6 Celermajer DS, Deanfield JE. Adults with congenital hear disease. BMF 1991;303:1413-14

7 Perloff JK. Congenital heart disease in adults: a new cardiovascular specialty. Circulation 1991;84:1881-90.

8 Somerville J, Webb GD, Skorton DJ, et al. Medical center experiences. 7 Am Coll Cardiol 1991;18:315-18.

9 Hunter S. Management of adults with congenital heart disease. Heart 1997;78:15.

10 Connelly MS, Webb GD, Somerville J, et al. Canadian consensus conference on adult congenital heart disease. Can $\mathcal{F}$ Cardiol 1998;14:395-452.

11 Engle MA, Adams FH, Betson C, et al. Resources for optimal long term care of congenital heart disease. Circulation mal long term care

12 Leonard H, O'Sullivan J, Hunter S. Family planning requirements in the adult congenital heart disease clinic. Heart 1996;76:60-2.
13 Presbitero P, Somerville J, Stone S, et al. Pregnancy in cyanotic congenital heart disease. Outcome of mother and fetus. Circulation 1994;89:2673-6.

14 Oakley CM. Pregnancy and congenital heart disease. Heart 1997;78:12-14.

15 Siu SC, Sermer M, Harrison DA, et al. Risk and predictors for pregnancy-related complications in women with heart disease. Circulation 1997;96:2789-94.

16 Allen HD, Gersony WM, Taubert KA. Insurability of the adolescent and young adult with heart
disease. Report from the Fifth Conference on Insurability, Oct 3-4, 1991, Columbus. Circulation 1992;86: bility, Oct

17 Dore A, Glancy L, Stone S, et al. Cardiac surgery for grown-up congenital heart patients: survey of $307 \mathrm{consecu-}$ tive operations from 1991 to 1994. Am f Cardiol 1997;80: 906-13.

18 Rosenberg HC, Webb G. Referral of young adult patients with congenital heart disease to adult centres. The Canadian Adult Congenital Heart Network. Can $\mathcal{F}$ Cardiol 1996;12:600-2. 